\title{
STRANGE QUARKS IN THE NUCLEON AND PARITY VIOLATION IN POLARIZED ELECTRON SCATTERING ${ }^{1}$
}

\author{
J. VAN DE WIELE ${ }^{(a)}$ and S. ONG $(a, b)$
}

(a) Institut de Physique Nucléaire/IN2P3, F-91406 Orsay Cedex, France

(b) Université de Picardie Jules Verne, Amiens, France

\begin{abstract}
In this review, we show that the measurement of asymmetry in polarized electronnucleon scattering provides information about the quark structure of the nucleon. The formalism of parity-violating electron-nucleon scattering with the theoretical assumptions is presented. An experimental overview of specific experiments with recent results as well as upcoming experiments is discussed.
\end{abstract}

\section{Introduction}

The structure of the nucleon at low energy is not well understood from the point of view of QCD. It is one of the most interesting (and difficult) questions in physics today.

It is commonly said that nucleons are bound states composed of three valence quarks. However, at high momentum transfer $Q^{2}$, the gluons and sea quark components of the nucleon are necessary to fit the deep inelastic scattering data. Certain decays of the $\phi$-meson suggest more strongly coupling of $\phi$ to the nucleon than expected on the basis of the OZI rule [1]; these results indicate a significant strange quark content in the nucleon. One possible explanation of the spin structure function data is that the proton contains a sizable polarized $\bar{s} s$ component.

It is not unreasonable to study the strange quark content of the nucleon and our probes to detect this presence are the following matrix elements :

a) $\left\langle p\left|\bar{\psi}_{s} \psi_{s}\right| p>\right.$ which is related to the sigma term in $\pi-N$ scattering, suggesting an unexpectedly signal for $\bar{s} s[2]$. The contribution of the strange quark is estimated to be about $10 \%$ of the mass of the proton [3].

b) $\left\langle p\left|\bar{\psi}_{s} \gamma_{\mu} \gamma_{5} \psi_{s}\right| p>\right.$ generated by spin structure data published by the EMC Collaboration in 1988 [4]. The $\bar{s} s$ pairs may play an important role in the spin structure functions intensively investigated at Hermes [5] and Compass [6].

c) $\left\langle p\left|\bar{\psi}_{s} \gamma_{\mu} \psi_{s}\right| p>\right.$ provides another observable in parity violating scattering of polarized electrons on nucleons. As discussed by Kaplan and Manohar [3], the study of neutral weak matrix elements can be used to determine the strange quark-antiquark matrix elements. From this matrix element, one can extracted informations on the strange quark contribution to the charge radius and magnetic moment of the proton. This is the purpose of the present study.

It is well known that the Standard Model $\left(S U(3)_{c} \otimes S U(2)_{L} \otimes U(1)_{Y}\right)$ provides a very good description of particles interactions. Here we are talking about strong, weak and electromagnetic interactions of known particles, essentially leptons and hadrons, the latter being strongly interacting particles built from quarks and antiquarks.

In this framework (SM), the exchange of intermediate gauge bosons $W^{ \pm}, Z$ with masses (approximatively 80 and $91 \mathrm{GeV} / \mathrm{c}^{2}$, respectively) and photon between particles, are responsible for various "charged and neutral currents" electro-weak interaction processes.

\footnotetext{
${ }^{1}$ Talk given at the INTERNATIONAL SCHOOL-WORKSHOP PRAHA-SPIN-2001, July 15-28, 2001, Prague, Czech Republic
} 
Neutral current effects should be present in the interaction of electrons with matter, but are then in competition with the electromagnetic interaction, which are normally much larger, as long as the center-of-mass energies and momenta remain small compared to the $\mathrm{W}^{ \pm}$or $\mathrm{Z}$ masses. However, at low or even moderately high energies, the large effects of electromagnetic interactions should prevent to detect the effects of the weak-neutral current interaction between electrons and hadrons through the interference term between electromagnetic and weak-neutral current amplitudes.

Weak interaction violates the discrete symmetry of parity, which exchanges the left and right orientations in space. The interference term reflects this feature and it is then crucial to measure the parity violating asymmetry in the cross section of elastic scattering of polarized electrons on unpolarized protons.

Such interference effects were indeed observed in a SLAC experiment in 1978 [7], in which a small asymmetry $\approx 10^{-4}$ in the deep-inelastic scattering of polarized electrons on deuterium could be measured.

The asymmetry measurement in elastic scattering of polarized electron beam will provide informations on the nucleon structure at low energy.

Our paper is organized as follows. In Sect. 2, the main aspects of electromagnetic form factors of the nucleon are emphasized. This allows one to introduce formalism and notations. The weak form factors are described in Sect. 3 using the formalism previously presented in Sect.2. An experimental overview is considered in sect. 4 and we summarize this report in Sect. 5.

\section{Electromagnetic form factors.}

In this section, we consider elastic ep scattering involving nucleon elastic form factors. The electromagnetic form factor of hadrons have still been the subject of theoretical and experimental studies. The coupling of elementary particle to the photon is determined by its charge and magnetic moment. For a composite particle, one includes the form factors which reflect the distribution of charge and current inside the particle.

We consider the elastic scattering of an electron on a proton (neutron) in initial state $|e>\equiv| e\left(P_{e}, s\right)>$ and $|p>\equiv| p(P, S)>(|n>\equiv| n(P, S)>)$ respectively and final states denoted by $\left|e^{\prime}>\equiv\right| e\left(P_{e^{\prime}}, s^{\prime}\right)>$ and $\left|p^{\prime}>\equiv\right| p\left(P^{\prime}, S^{\prime}\right)>\left(\left|n^{\prime}>\equiv\right| n\left(P^{\prime}, S^{\prime}\right)>\right)$ respectively. The four-momentum transfer is written $q^{\mu}=P_{e}^{\mu}-P_{e^{\prime}}^{\mu}$. As we neglect the electron mass, the expressions of the momentum transfer and the energy of the outgoing electron are simple:

$$
\begin{array}{r}
Q^{2} \equiv-q^{2}=-\left(P_{e}-P_{e^{\prime}}\right)^{2}=4 E_{e} E_{e^{\prime}} \sin ^{2}\left(\theta_{e^{\prime}} / 2\right) \\
E_{e^{\prime}}=\frac{E_{e}}{1+2 E_{e} / M \sin ^{2}\left(\theta_{e^{\prime}} / 2\right)}
\end{array}
$$

where $M$ is the nucleon mass, $E_{e}\left(E_{e^{\prime}}\right)$ is the incident energy of the incoming (outgoing) electron and $\theta_{e^{\prime}}$ is the scattering angle in the laboratory. The transition amplitude associated to the exchange of a photon and which corresponds to the Feynman diagram of Fig. 1 is the product of the leptonic and hadronic currents:

$$
\mathcal{T}^{E M}\left(p^{\prime} e^{\prime}, e p\right)=-\frac{4 \pi \alpha}{q^{2}} Q_{e} J_{\mu}^{e} J^{p} E M \mu
$$

where $Q_{e}=-1$ is the charge of the electron in unit of $e$ and $\alpha=1 / 137$ is the fine-structure constant. The leptonic current is given by: 


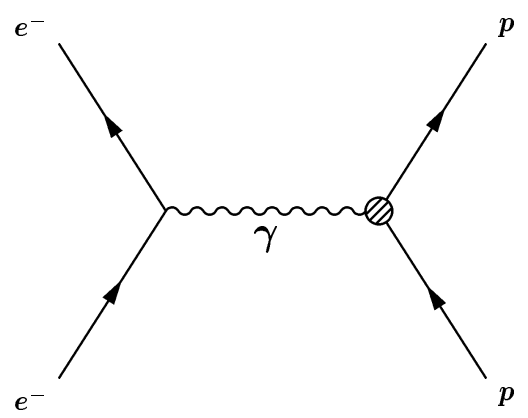

Figure 1: Feynman diagram:photon exchange.

$$
j_{\mu}^{e}=\left\langle e^{\prime}\left|\hat{\jmath}_{\mu}^{e}\right| e>=\overline{\mathcal{u}}_{e^{\prime}} \gamma_{\mu} \mathcal{U}_{e}\right.
$$

The most general possible hadronic current for elastic electron proton scattering assuming current conservation, Lorentz invariance and time reversal is

$$
J^{p} E M \mu=<p^{\prime}\left|\hat{J}^{E M \mu}\right| p>=\overline{\mathcal{u}}_{p^{\prime}}\left[F_{1}^{p, \gamma}\left(Q^{2}\right) \gamma^{\mu}+i \frac{F_{2}^{p, \gamma}\left(Q^{2}\right)}{2 M} \sigma^{\mu \nu} q_{\nu}\right] \mathcal{u}_{p}
$$

where $F_{1}^{p, \gamma}\left(Q^{2}\right)$ and $F_{2}^{p, \gamma}\left(Q^{2}\right)$ are the Dirac and Pauli electromagnetic form factors respectively and $\mathcal{u}_{p}, \overline{\mathcal{u}}_{p^{\prime}}$ are the proton four component Dirac spinor and it adjoint. Formulas for cross sections are usually expressed in terms of the Sachs form factors defined by:

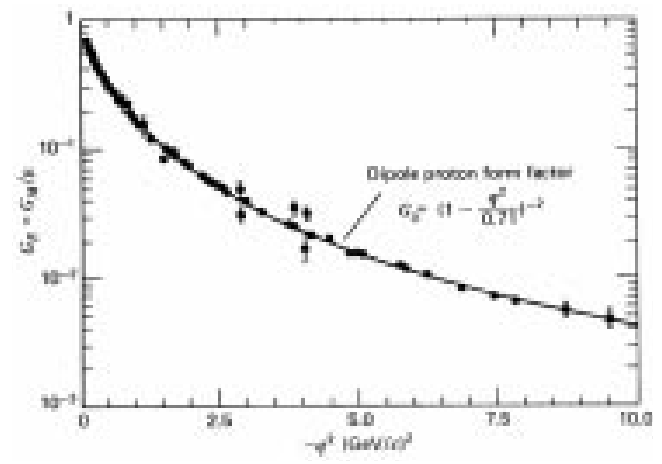

Figure 2: The proton electric form factor as a function of $q^{2}$.

$$
G_{E}^{p, \gamma}\left(Q^{2}\right)=F_{1}^{p, \gamma}\left(Q^{2}\right)-\tau F_{2}^{p, \gamma}\left(Q^{2}\right) \quad G_{M}^{p, \gamma}\left(Q^{2}\right)=F_{1}^{p, \gamma}\left(Q^{2}\right)+F_{2}^{p, \gamma}\left(Q^{2}\right)
$$

where $\tau=Q^{2} / 4 M^{2}$. These electric $G_{E}$ and magnetic $G_{M}$ form factors are normalized such as:

$$
F_{1}^{p, \gamma}(0)=1 \quad F_{2}^{p, \gamma}(0)=\mu_{p}-1=\kappa_{p} \quad G_{E}^{p, \gamma}(0)=1 \quad G_{M}^{p, \gamma}(0)=\mu_{p}
$$

where $\mu_{p}=2.793$ is the electromagnetic magnetic moment of the proton and $\kappa_{p}$ its anomalous magnetic moment. 


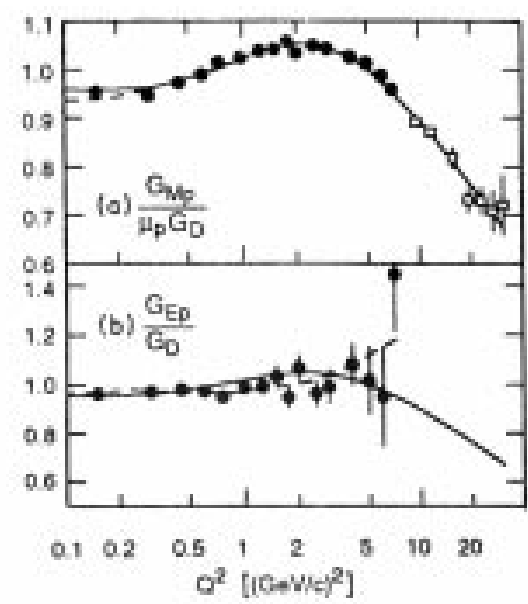

Figure 3: Proton magnetic and electric form factors.

For an arbitrary value of $Q^{2} \neq 0$, one needs to assume the $Q^{2}$-dependence of these form factors. A simple parametrization known as the dipole fit is used for the electric form factor of the proton [8] (Fig. 2):

$$
G_{E}^{p, \gamma}\left(Q^{2}\right) \simeq G_{D}\left(Q^{2}\right)=\left(1+Q^{2} / 0.71\right)^{-2}
$$

In the measured $Q^{2}$ range of our interest, form factor scaling $G_{E}^{p, \gamma}\left(Q^{2}\right) \sim G_{M}^{p, \gamma}\left(Q^{2}\right) / \mu_{p}$ seems to work reasonably well [9] (Fig. 3) and this assumption is used in this study.

The electric radii of the nucleon given by the slope of the associated electric form factor at $Q^{2}=0$.

$$
<r^{2}>=-\left.6 \frac{\partial G_{E}\left(Q^{2}\right)}{\partial Q^{2}}\right|_{Q^{2}=0},
$$

and the magnetic moment $\mu_{p}=G_{M}\left(Q^{2}=0\right)$ are the physical observables related to these form factors.

The differential cross section of $e p$ elastic scattering can be written as :

$$
\begin{aligned}
\frac{d \sigma^{p, \gamma}}{d \Omega} & =\left(\frac{d \sigma}{d \Omega}\right)_{\text {Mott }} \frac{E_{e^{\prime}}}{E_{e}}\left[\frac{\left(G_{E}^{p, \gamma}\right)^{2}+\tau\left(G_{M}^{p, \gamma}\right)^{2}}{1+\tau}+2 \tau\left(G_{M}^{p, \gamma}\right)^{2} \tan ^{2}\left(\theta_{e^{\prime}} / 2\right)\right] \\
& =\left(\frac{d \sigma}{d \Omega}\right)_{\text {Mott }} \frac{E_{e^{\prime}}}{E_{e}} \frac{1}{\varepsilon(1+\tau)}\left[\varepsilon\left(G_{E}^{p, \gamma}\right)^{2}+\tau\left(G_{M}^{p, \gamma}\right)^{2}\right]
\end{aligned}
$$

with

$$
\left(\frac{d \sigma}{d \Omega}\right)_{\mathrm{Mott}}=\frac{\alpha^{2} \cos ^{2}\left(\theta_{e^{\prime}} / 2\right)}{4 E_{e}^{2} \sin ^{4}\left(\theta_{e^{\prime}} / 2\right)}
$$

and:

$$
\varepsilon=\frac{1}{1+2(1+\tau) \tan ^{2} \theta_{e^{\prime}} / 2}
$$

is the transverse polarization of the virtual photon exchanged. 
It is clear from eq.9 and eq.10 that if one measures the elastic cross section at one angle $\theta_{e^{\prime}}$ and for one incident energy $E_{e}$, one gets only a linear combination of the electric and magnetic form factors. It is possible to determine completely these form factors if we choose, for a value of $Q^{2}$ fixed, another kinematic set defined by other values of $E_{e}$ and $\theta_{e^{\prime}}$. This procedure is known as the Rosenbluth separation.

The neutron cross section is given by changing the superscript $p$ to $n$. The neutron form factors are normalized such as:

$$
\begin{gathered}
F_{1}^{n, \gamma}(0)=0 \quad F_{2}^{n, \gamma}(0)=\mu_{n}=\kappa_{n} \\
G_{E}^{n, \gamma}(0)=0 \quad G_{M}^{n, \gamma}(0)=\mu_{n}=-1.913
\end{gathered}
$$

Form factors of the neutron are difficult to measure [10,11, 12, 13, 14]. Most of the available data come from the quasi-elastic scattering from deuterons (Fig. 4). Here again, it was observed that:

$$
G_{M}^{n, \gamma}\left(Q^{2}\right) \simeq \mu_{n} G_{D}\left(Q^{2}\right)
$$

Precision measurement of $G_{E}^{n, \gamma}$ is required in order to extract the strange form factor without additional errors. The Galster parametrization [15] seems to work for the electric form factor of the neutron:

$$
G_{E}^{n, \gamma}\left(Q^{2}\right)=-\mu_{n} \tau G_{D}\left(Q^{2}\right) /(1+5.6 \tau)
$$

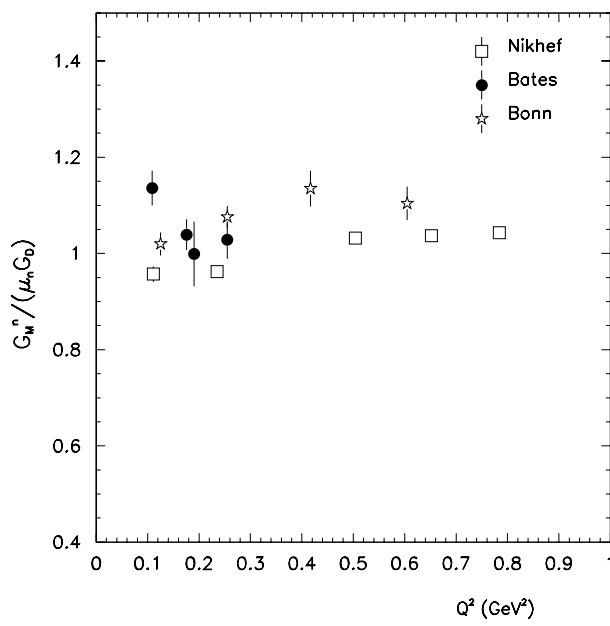

a)

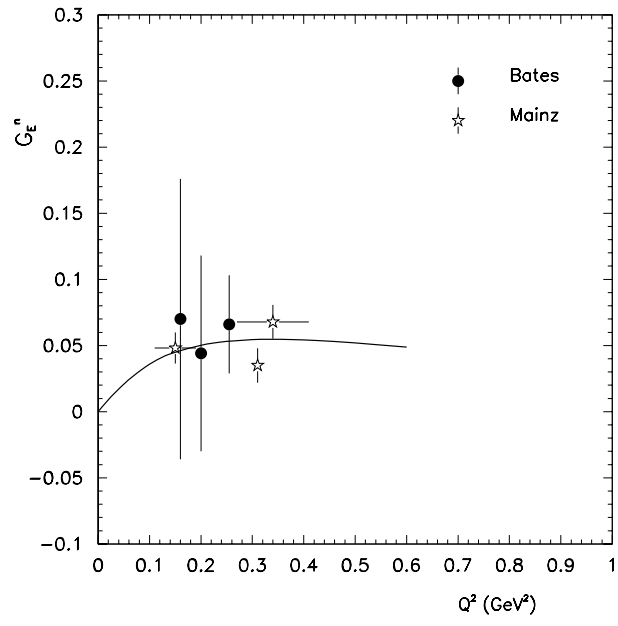

b)

Figure 4: Neutron magnetic a) and electric b) form factors. 


\begin{tabular}{ccrrr}
\hline fermion & $g_{V}^{f}$ & $g_{A}^{f}$ & $Q_{f}$ & $T_{3}^{f}$ \\
\hline$\nu_{e}$ & 1 & -1 & 0 & $\frac{1}{2}$ \\
$e^{-}$ & $-1+4 \sin ^{2} \theta_{W}$ & 1 & -1 & $-\frac{1}{2}$ \\
$\nu_{\mu}$ & 1 & -1 & 0 & $\frac{1}{2}$ \\
$\mu^{-}$ & $-1+4 \sin ^{2} \theta_{W}$ & 1 & -1 & $-\frac{1}{2}$ \\
$u$ & $1-\frac{8}{3} \sin ^{2} \theta_{W}$ & -1 & $\frac{2}{3}$ & $\frac{1}{2}$ \\
$d$ & $-1+\frac{4}{3} \sin ^{2} \theta_{W}$ & 1 & $-\frac{1}{3}$ & $-\frac{1}{2}$ \\
$s$ & $-1+\frac{4}{3} \sin ^{2} \theta_{W}$ & 1 & $-\frac{1}{3}$ & $-\frac{1}{2}$ \\
\hline
\end{tabular}

Table 1: Electroweak coupling of charged fundamental particles and the third component of the weak isospin.

\section{$3 \quad$ Weak form factors in parity violating ep scattering}

\subsection{Formalism}

The Standard Model (SM) is based on a gauge field theory with a symmetry group $S U(3)_{c} \otimes$ $S U(2)_{L} \otimes U(1)_{Y}$. There are 12 fundamental gauge fields and 3 gauge couplings and, for matter fields, three families of quarks and leptons with the fundamental couplings to the photon and $\mathrm{Z}^{0}$ respectively as :

$$
i e Q_{f} \gamma_{\mu}, \quad \frac{i g}{4 \cos \theta_{W}} \gamma_{\mu}\left(g_{V}^{f}+g_{A}^{f} \gamma_{5}\right)
$$

Where $e, g$ are the electromagnetic and weak coupling strengths and $\theta_{W}$ is the weak mixing angle. The three accurate input parameters of the SM are the fine structure constant $\alpha$, the Fermi constant $G_{F}$ and the $\mathrm{Z}^{0}$-mass $M_{Z}$. At tree level, one can express the weak mixing angle as a function of these input parameters through the relation :

$$
\sin ^{2} \theta_{W} \cos ^{2} \theta_{W}=\frac{\pi \alpha}{\sqrt{2} G_{F} M_{Z}^{2}}
$$

The electromagnetic charge of the fermion $Q_{f}$ and the vector and axial "charge" defining in formula (17) are given by :

$$
\begin{array}{r}
g_{V}^{f}=2 T_{3}^{f}-4 Q_{f} \sin ^{2} \theta_{W} \\
g_{A}^{f}=-2 T_{3}^{f}
\end{array}
$$

We summarize in table 1 , the electroweak coupling of particles and the third component $T_{3}^{f}$ of the weak isodoublets.

Let us consider now, the elastic electroweak scattering (Fig.5) where the weak neutral current corresponding to the $Z^{0}$ exchange is given, in order to respect the formalism considered, in the following form :

$$
j_{\mu 5}^{e}=\overline{\mathcal{u}}_{e^{\prime}} \gamma_{\mu} \gamma_{5} \mathcal{U}_{e}
$$




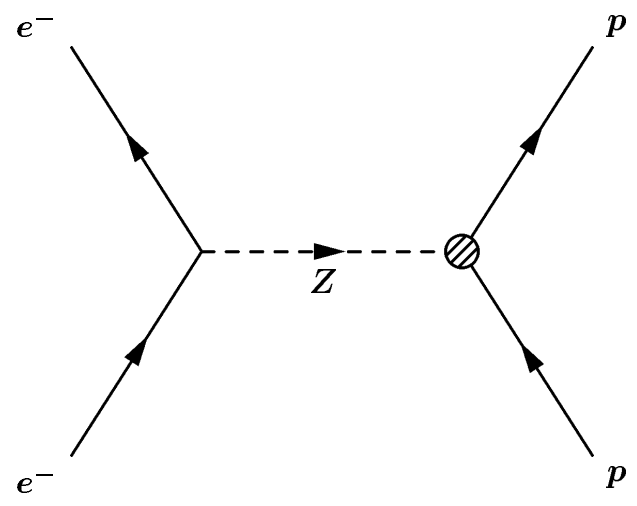

Figure 5: Feynman diagram: $Z^{0}$ exchange.

is the axial electromagnetic current at the electron vertex, the vector part is already defined in section 2.

The hadronic vector current associated to the exchange of $Z^{0}$, reads

$$
J^{p} N C \mu=<p^{\prime}\left|\hat{J}^{N C \mu}\right| p>=\bar{u}_{p^{\prime}}\left[\widetilde{F}_{1}^{p} \gamma^{\mu}+i \frac{\widetilde{F}_{2}^{p}}{2 M} \sigma^{\mu \nu} q_{\nu}\right] \mathcal{u}_{p}
$$

where $\widetilde{F}_{1}^{p}$ and $\widetilde{F}_{2}^{p}$ are the neutral vector form factors.

And the hadronic axial neutral current is expanded as following:

$$
J^{p} N C \mu 5=<p^{\prime}\left|\hat{J}^{N C \mu 5}\right| p>=\overline{\mathcal{u}}_{p^{\prime}}\left[\widetilde{G}_{A}^{p} \gamma^{\mu}+i \frac{\widetilde{G}_{P}^{p}}{M} q^{\mu}\right] \gamma^{5} u_{p}
$$

where $\widetilde{G}_{A}^{p}$ and $\widetilde{G}_{P}^{p}$ are the axial-vector and pseudo-scalar form factors respectively. This latter $\widetilde{G}_{P}^{p}$ form factor does not contribute in parity violation electron scattering to leading order in electroweak couplings and will be neglected in the following part.

Many form factors are necessary in this formalism. However, we can relate all the form factors to a few flavor form factors. First, we expand the current operators - electromagnetic, vector and axial-vector - in terms of the corresponding quark current operators:

$$
\begin{aligned}
\hat{J}_{\mu}^{E M} & =\sum_{f=u, d, s} Q_{f} \bar{\psi}_{f} \gamma_{\mu} \psi_{f} \quad \hat{J}_{\mu}^{N C}=\sum_{f=u, d, s} g_{V}^{f} \bar{\psi}_{f} \gamma_{\mu} \psi_{f} \\
\hat{J}_{\mu 5}^{N C} & =\sum_{f=u, d, s} g_{A}^{f} \bar{\psi}_{f} \gamma_{\mu} \gamma_{5} \psi_{f}
\end{aligned}
$$

where the expansion is restricted to the $S U(3)_{F}$ flavor space. The physical current are the matrix elements of these operators between the initial $\mid p>(\mid n>)$ and final states $\mid p^{\prime}>$ $\left(\left|n^{\prime}\right\rangle\right)$. We must evaluate the matrix elements $\left\langle x^{\prime}\left|\bar{\psi}_{f} \gamma_{\mu} \psi_{f}\right| x\right\rangle$ and $\left\langle x^{\prime}\left|\bar{\psi}_{f} \gamma_{\mu} \gamma_{5} \psi_{f}\right| x\right\rangle$ where $x$ is a proton $p$ or a neutron $n$. At this stage of theoretical development we still do not have a reliable method for calculating such a matrix element. We then define the flavor form factors:

$$
<x^{\prime}\left|\bar{\psi}_{f} \gamma_{\mu} \psi_{f}\right| x>\equiv \overline{\mathcal{u}}_{x^{\prime}}\left(F_{1}^{f, x} \gamma_{\mu}+i \frac{F_{2}^{f, x}}{2 M} \sigma_{\mu \nu} q^{\nu}\right) \mathcal{u}_{x}
$$




$$
<x^{\prime}\left|\bar{\psi}_{f} \gamma_{\mu} \gamma_{5} \psi_{f}\right| x>\equiv \overline{\mathcal{u}}_{x^{\prime}}\left(\widetilde{G}_{A}^{f, x} \gamma_{\mu}+i \frac{\widetilde{G}_{P}^{f, x}}{M} q_{\mu}\right) \gamma_{5} \mathcal{u}_{x}
$$

As explained before, we ignore the $\widetilde{G}_{P}^{f, x}$ form factors. There then are a total of eighteen form factors. This number of form factors is reduced if we assume the charge symmetry between protons and neutrons (isospin symmetry).

$$
\begin{gathered}
F_{1}^{u} \equiv F_{1}^{u, p}=F_{1}^{d, n} \quad F_{2}^{u} \equiv F_{2}^{u, p}=F_{2}^{d, n} \\
F_{1}^{d} \equiv F_{1}^{d, p}=F_{1}^{u, n} \quad F_{2}^{d} \equiv F_{2}^{d, p}=F_{2}^{u, n} \\
F_{1}^{s} \equiv F_{1}^{s, p}=F_{1}^{s, n} \quad F_{2}^{s} \equiv F_{2}^{s, p}=F_{2}^{s, n} \\
G_{A}^{u} \equiv \widetilde{G}_{A}^{u, p}=\widetilde{G}_{A}^{d, n} \quad G_{A}^{d} \equiv \widetilde{G}_{A}^{d, p}=\widetilde{G}_{A}^{u, n} \quad G_{A}^{s} \equiv \widetilde{G}_{A}^{s, p}=\widetilde{G}_{A}^{s, n}
\end{gathered}
$$

Within this approximation, the electromagnetic vector current form factors and the neutral vector current form factors are expressed as a linear combination of the quark form factors:

$$
\begin{aligned}
F_{1}^{p, \gamma} & =\frac{2}{3} F_{1}^{u}-\frac{1}{3} F_{1}^{d}-\frac{1}{3} F_{1}^{s} \quad F_{2}^{p, \gamma}=\frac{2}{3} F_{2}^{u}-\frac{1}{3} F_{2}^{d}-\frac{1}{3} F_{2}^{s} \\
F_{1}^{n, \gamma}= & \frac{2}{3} F_{1}^{d}-\frac{1}{3} F_{1}^{u}-\frac{1}{3} F_{1}^{s} \quad F_{2}^{n, \gamma}=\frac{2}{3} F_{2}^{d}-\frac{1}{3} F_{2}^{u}-\frac{1}{3} F_{2}^{s} \\
\widetilde{F}_{1}^{p} & =\left(1-\frac{8}{3} \sin ^{2} \theta_{W}\right) F_{1}^{u}-\left(1-\frac{4}{3} \sin ^{2} \theta_{W}\right)\left(F_{1}^{d}+F_{1}^{s}\right) \\
\widetilde{F}_{2}^{p} & =\left(1-\frac{8}{3} \sin ^{2} \theta_{W}\right) F_{2}^{u}-\left(1-\frac{4}{3} \sin ^{2} \theta_{W}\right)\left(F_{2}^{d}+F_{2}^{s}\right) \\
\widetilde{F}_{1}^{n} & =\left(1-\frac{8}{3} \sin ^{2} \theta_{W}\right) F_{1}^{d}-\left(1-\frac{4}{3} \sin ^{2} \theta_{W}\right)\left(F_{1}^{u}+F_{1}^{s}\right) \\
\widetilde{F}_{2}^{n} & =\left(1-\frac{8}{3} \sin ^{2} \theta_{W}\right) F_{2}^{d}-\left(1-\frac{4}{3} \sin ^{2} \theta_{W}\right)\left(F_{2}^{u}+F_{2}^{s}\right)
\end{aligned}
$$

Generally the different observables are expressed in terms of the Sachs form factors defined as usual by

$$
\widetilde{G}_{E}^{p}\left(Q^{2}\right)=\widetilde{F}_{1}^{p}\left(Q^{2}\right)-\tau \widetilde{F}_{2}^{p}\left(Q^{2}\right) \quad \widetilde{G}_{M}^{p}\left(Q^{2}\right)=\widetilde{F}_{1}^{p}\left(Q^{2}\right)+\widetilde{F}_{2}^{p}\left(Q^{2}\right)
$$

As we will show in the next subsection, the neutral vector current form factors which enter explicitly in the expression of the asymmetry for the proton are

$$
G_{E}^{p, Z}=\frac{\widetilde{G}_{E}^{p}}{4} \quad G_{M}^{p, Z}=\frac{\widetilde{G}_{M}^{p}}{4}
$$

and for the neutron

$$
G_{E}^{n, Z}=\frac{\widetilde{G}_{E}^{n}}{4} \quad G_{M}^{n, Z}=\frac{\widetilde{G}_{M}^{n}}{4}
$$


Using the equations (32-37) it is possible to express the form factor associated to the $u$ and $d$ quarks in terms of the electromagnetic proton and neutron form factors and the $s$ quark vector current form factor.

$$
\begin{aligned}
& G_{E}^{p, Z}\left(Q^{2}\right)=\frac{\xi_{V}^{p} G_{E}^{p, \gamma}+\xi_{V}^{n} G_{E}^{n, \gamma}+\xi_{V}^{(0)} G_{E}^{s}}{4}, \quad G_{M}^{p, Z}\left(Q^{2}\right)=\frac{\xi_{V}^{p} G_{M}^{p, \gamma}+\xi_{V}^{n} G_{M}^{n, \gamma}+\xi_{V}^{(0)} G_{M}^{s}}{4} \\
& G_{E}^{n, Z}\left(Q^{2}\right)=\frac{\xi_{V}^{n} G_{E}^{p, \gamma}+\xi_{V}^{p} G_{E}^{n, \gamma}+\xi_{V}^{(0)} G_{E}^{s}}{4}, \quad G_{M}^{n, Z}\left(Q^{2}\right)=\frac{\xi_{V}^{n} G_{M}^{p, \gamma}+\xi_{V}^{p} G_{M}^{n, \gamma}+\xi_{V}^{(0)} G_{M}^{s}}{4}
\end{aligned}
$$

where

$$
G_{E}^{s}\left(Q^{2}\right)=F_{1}^{s}\left(Q^{2}\right)-\tau F_{2}^{s}\left(Q^{2}\right) \quad G_{M}^{s}\left(Q^{2}\right)=F_{1}^{s}\left(Q^{2}\right)+F_{2}^{s}\left(Q^{2}\right)
$$

If the radiative corrections are neglected, the expressions of the coefficients $\xi_{V}^{p}, \xi_{V}^{n}, \xi_{\mathrm{V}}^{(0)}$ are given by Musolf et al [16].

$$
\xi_{V}^{p}=1-4 \sin ^{2} \theta_{W} \quad \xi_{V}^{n}=-1 \quad \xi_{V}^{(0)}=-1
$$

The axial-vector part in the expression of the asymmetry is written:

$$
G_{A}^{p, Z} \equiv \frac{\widetilde{G}_{A}^{p}}{2} \quad G_{A}^{n, Z} \equiv \frac{\widetilde{G}_{A}^{n}}{2}
$$

where $\widetilde{G}_{A}^{p}$ and $\widetilde{G}_{A}^{n}$ are the axial-vector form factors defined by Eq. (23). Then, we obtain

$$
\begin{aligned}
& G_{A}^{p, Z}\left(Q^{2}\right)=\frac{1}{2}\left[G_{A}^{d}\left(Q^{2}\right)-G_{A}^{u}\left(Q^{2}\right)\right]+\frac{G_{A}^{s}\left(Q^{2}\right)}{2} \\
& G_{A}^{n, Z}\left(Q^{2}\right)=\frac{1}{2}\left[G_{A}^{u}\left(Q^{2}\right)-G_{A}^{d}\left(Q^{2}\right)\right]+\frac{G_{A}^{s}\left(Q^{2}\right)}{2}
\end{aligned}
$$

When $Q^{2}=0, G_{A}^{q}(0)=\Delta q$ is the contribution of the $q$-quark and $\bar{q}$-quark to the spin of the proton.

$$
G_{A}^{u}(0)=\Delta u \quad G_{A}^{d}(0)=\Delta d \quad G_{A}^{s}(0)=\Delta s
$$

The spin dependent structure function $g_{1}(x)$ of the nucleon has been measured in deepinelastic lepton scattering experiments. In first order, the first moment of the proton ${ }_{1}^{p} \equiv$ $\int_{0}^{1} g_{1}(x) d x$ is related to $\Delta u, \Delta d, \Delta s$ by

$$
{ }_{1}^{p}=\frac{1}{2}\left[\frac{4}{9} \Delta u+\frac{1}{9} \Delta d+\frac{1}{9} \Delta s\right]
$$

The difference $\Delta u-\Delta d=g_{A}=1.26=F+D$ is known from neutron beta decay [17] and using the results of hyperon beta decays [18] $\Delta u+\Delta d-2 \Delta s=3 F-D \approx-0.60$. The combination of the three experiments allows to obtain the individual flavor components $\Delta u$, $\Delta d$ and $\Delta s$ in the proton. They agree with the calculations based on various assumptions [19]. We give here the results based on $S U(3)_{F}: \Delta u \approx 0.82, \Delta d \approx-0.44$, and $\Delta s \approx-0.11$. 
The expressions given above are obtained within the tree level approximation. Radiative corrections may modify these expressions. It is not the subject of this talk to discuss them in detail. To calculate accurately these radiative corrections is a very difficult task. Some Feynman diagrams taking into account such corrections are displayed in Fig.6. The first corrections occur at the quark level but it is also possible to consider the interaction of low momentum virtual $s \bar{s}$ pair with valence quarks to form strange hadronic intermediate states $(K, \Lambda, \phi, \ldots)$. The distinction between the considered processes is not rigorous, there may be some double counting, missing contributions and trouble with gauge invariance.

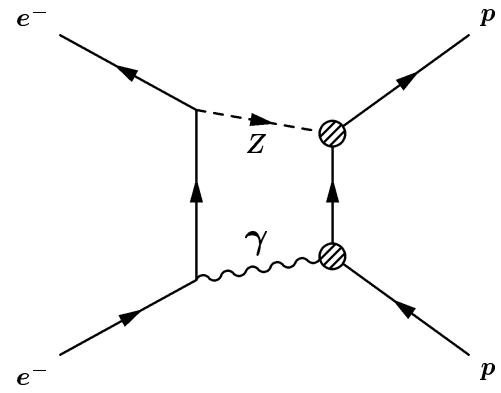

a)

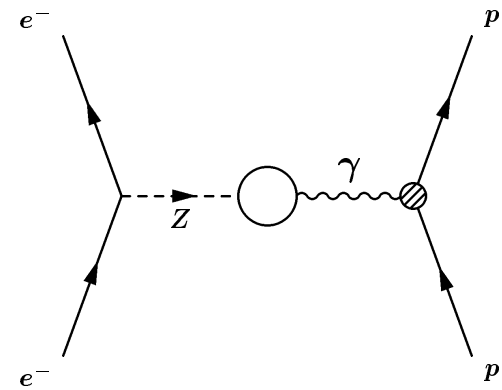

b)

Figure 6: Examples of radiative corrections

To obtain the expressions taking into account the radiative corrections, it is convenient to expand the current operators in terms of the $S U(3)_{F}$ octet and singlet currents. We have:

$$
\begin{aligned}
\xi_{V}^{p} & =\left(1-4 \sin ^{2} \theta_{W}\right)\left(1+R_{V}^{p}\right) \quad \xi_{V}^{n}=-\left(1+R_{V}^{n}\right) \\
\xi_{V}^{(0)} & =-\left(1+R_{V}^{(0)}\right)
\end{aligned}
$$

where $R_{V}^{p}$ and $R_{V}^{n}$ are linear combination of isoscalar and isovector corrections. If we take the values of [16], we have

$$
R_{\mathrm{V}}^{p}=-0.0529 \quad R_{\mathrm{V}}^{n}=-0.0144 \quad R_{\mathrm{V}}^{(0)} \approx 0
$$

In agreement with these values, it is believed that the radiative corrections for the terms involving the vector form factors are small and well known.

The radiative corrections to the axial proton current in electron scattering are substantial. To the single quark radiative corrections, some additional terms have been computed on the basis of an effective field theory. They correspond to the anapole moment of the proton which is the effect of the parity-violating coupling of the photon to the proton.

According to Musolf et al.[16], the axial form factor $G_{A}^{p, Z}$ and $G_{A}^{n, Z}$ are decomposed as:

$$
\begin{gathered}
G_{A}^{p, Z}\left(Q^{2}\right)=-\left(1+R_{A}^{T=1}\right) G_{A}^{(3)}\left(Q^{2}\right)+\sqrt{3} R_{A}^{T=0} \frac{G_{A}^{(8)}\left(Q^{2}\right)}{2}+\xi_{A}^{s} \frac{G_{A}^{s}\left(Q^{2}\right)}{2} \\
G_{A}^{n, Z}\left(Q^{2}\right)=\left(1+R_{A}^{T=1}\right) G_{A}^{(3)}\left(Q^{2}\right)+\sqrt{3} R_{A}^{T=0} \frac{G_{A}^{(8)}\left(Q^{2}\right)}{2}+\xi_{A}^{s} \frac{G_{A}^{s}\left(Q^{2}\right)}{2}
\end{gathered}
$$


where

$$
G_{A}^{(3)}\left(Q^{2}\right)=\frac{G_{A}^{u}\left(Q^{2}\right)-G_{A}^{d}\left(Q^{2}\right)}{2} \quad G_{A}^{(8)}(0)=\frac{1}{2 \sqrt{3}}(3 F-D)
$$

and

$$
\xi_{A}^{s}=1+R_{A}^{(0)}
$$

At $Q^{2}=0$, if we choose $\sqrt{3} R_{A}^{T=0} \approx-0.43$ [16], we have

$$
G_{A}^{(3)}(0)=\frac{g_{A}}{2}=0.63 \quad \sqrt{3} R_{A}^{T=0} G_{A}^{(8)}(0) \approx 0.074
$$

this last value is in agreement with a recent publication by Zhu et al. [20] These authors find for $R_{A}^{T=1}$ the value -0.41 , which is not very different from Musolf et al. [16]

We now define the isovector and isoscalar non strange axial form factor:

$$
\begin{array}{r}
G_{A}^{T=1}\left(Q^{2}\right) \equiv-2\left(1+R_{A}^{T=1}\right) G_{A}^{(3)}\left(Q^{2}\right) \\
G_{A}^{T=0}\left(Q^{2}\right) \equiv \sqrt{3} R_{A}^{T=0} G_{A}^{(8)}\left(Q^{2}\right)
\end{array}
$$

The numerical values obtained in the equation (57) show that the isoscalar axial form factor is small compared to the isovector axial form factor.

Finally we write

$$
\begin{gathered}
G_{A}^{p, Z}\left(Q^{2}\right)=\frac{1}{2}\left[G_{A}^{T=1}\left(Q^{2}\right)+G_{A}^{T=0}\left(Q^{2}\right)+\xi_{A}^{s} G_{A}^{s}\left(Q^{2}\right)\right] \\
G_{A}^{n, Z}\left(Q^{2}\right)=\frac{1}{2}\left[-G_{A}^{T=1}\left(Q^{2}\right)+G_{A}^{T=0}\left(Q^{2}\right)+\xi_{A}^{s} G_{A}^{s}\left(Q^{2}\right)\right]
\end{gathered}
$$

The estimation of these terms has been considered assuming a parameterized dependence in $Q^{2}$ as:

$$
G_{A}^{f}\left(Q^{2}\right)=G_{A}^{f}(0) G_{D}^{A}\left(Q^{2}\right), \quad G_{D}^{A}\left(Q^{2}\right)=\frac{1}{\left(1+\frac{Q^{2}}{M_{A}^{2}}\right)^{2}}=\frac{1}{\left(1+\lambda_{D}^{A} \tau\right)^{2}}
$$

where $f=u, d, s$ and $M_{A} \approx 1.032 \mathrm{GeV}$.

The axial strange form factor reads:

$$
G_{A}^{s}=\Delta s G_{D}^{A} \xi_{A}^{(s)} \quad \xi_{A}^{(s)}=\frac{1}{1+\lambda_{A}^{(s)} \tau}
$$

\subsection{Elastic Electroweak Scattering and Strange form factors}

The transition amplitude of $e p$ elastic electroweak scattering is the sum of the electromagnetic amplitude (EM) due to the exchange of a virtual photon and of the neutral current (NC) amplitude due to the exchange of the $Z^{0}$.

$$
\mathcal{T}\left(p^{\prime} e^{\prime}, e p\right)=\mathcal{T}^{E M}\left(p^{\prime} e^{\prime}, e p\right)+\mathcal{T}^{N C}\left(p^{\prime} e^{\prime}, e p\right)
$$

where

$$
\mathcal{T}^{N C}\left(p^{\prime} e^{\prime}, e p\right)=\frac{-G_{F}}{2 \sqrt{2}}\left(g_{V}^{e} j_{\mu}^{e}+g_{A}^{e} j_{\mu 5}^{e}\right)\left(J^{p} N C \mu+J^{p} N C \mu 5\right)
$$


The term in the amplitude which violates the parity is given by

$$
\mathcal{T}^{P V}\left(p^{\prime} e^{\prime}, e p\right)=\frac{-G_{F}}{2 \sqrt{2}}\left(g_{V}^{e} j_{\mu}^{e} J^{p} N C \mu 5+g_{A}^{e} j_{\mu 5}^{e} J^{p} N C \mu\right)
$$

The neutral current amplitude $\mathcal{T}^{N C}\left(p^{\prime} e^{\prime}, e p\right)$ in Eq. 64 is very small, compared to the electromagnetic amplitude $\mathcal{T}^{E M}\left(p^{\prime} e^{\prime}, e p\right)$, so the measurement of the elastic cross section which is proportional to the square of the total amplitude does not help very much. A polarized longitudinal electron beam is an efficient tool to measure the interference between the electromagnetic and the neutral amplitude. If we measure the asymmetry defined as

$$
A_{L R}=\frac{\sigma_{+}-\sigma_{-}}{\sigma_{+}+\sigma_{-}}
$$

where $\sigma_{+}$is the cross section associated with incident electron with an helicity equal to $1 / 2$ and $\sigma_{-}$is the cross section associated with incident electron with an helicity equal to $-1 / 2$. The expression of the asymmetry on the proton is given by:

$$
A_{L R}^{p}=-\frac{G_{F} Q^{2}}{\pi \alpha \sqrt{2}} \frac{1}{\sigma_{p}}\left[\varepsilon G_{E}^{p, \gamma} G_{E}^{p, Z}+\tau G_{M}^{p, \gamma} G_{M}^{p, Z}-\frac{1-4 \sin ^{2} \theta_{W}}{2} \varepsilon^{\prime} G_{M}^{p, \gamma} G_{A}^{p, Z}\right]
$$

where

$$
\varepsilon^{\prime}=\sqrt{\left(1-\varepsilon^{2}\right) \tau(1+\tau)}
$$

and

$$
\begin{array}{r}
\sigma_{p}=\varepsilon\left(G_{E}^{p, \gamma}\right)^{2}+\tau\left(G_{M}^{p, \gamma}\right)^{2} \\
G_{E}^{p, Z}=\frac{\widetilde{G}_{E}^{p}}{4}, \quad G_{M}^{p, Z}=\frac{\widetilde{G}_{M}^{p}}{4}, \quad G_{A}^{p, Z}=\frac{\widetilde{G}_{A}^{p}}{2}
\end{array}
$$

The expression for the asymmetry in the electron scattering on the neutron is very similar to the proton case:

$$
A_{L R}^{n}=-\frac{G_{F} Q^{2}}{\pi \alpha \sqrt{2}} \frac{1}{\sigma_{n}}\left[\varepsilon G_{E}^{n, \gamma} G_{E}^{n, Z}+\tau G_{M}^{n, \gamma} G_{M}^{n, Z}-\frac{1-4 \sin ^{2} \theta_{W}}{2} \varepsilon^{\prime} G_{M}^{n, \gamma} G_{A}^{n, Z}\right]
$$

where

$$
\begin{array}{r}
\sigma_{n}=\varepsilon\left(G_{E}^{n, \gamma}\right)^{2}+\tau\left(G_{M}^{n, \gamma}\right)^{2} \\
G_{E}^{n, Z}=\frac{\widetilde{G}_{E}^{n}}{4}, \quad G_{M}^{n, Z}=\frac{\widetilde{G}_{M}^{n}}{4}, \quad G_{A}^{n, Z}=\frac{\widetilde{G}_{A}^{n}}{2}
\end{array}
$$

The proton asymmetry is the sum of the term $A_{L R}^{p, 0}$ without any strange form factor and of the term $A_{L R}^{p, s}$ which contains all the strange form factors:

$$
\begin{gathered}
A_{L R}^{p}=A_{L R}^{p, 0}+A_{L R}^{p, s} \\
A_{L R}^{p, 0}=-\frac{G_{F} Q^{2}}{\pi \alpha \sqrt{2}} \frac{1}{4 \sigma_{p}}\left[\xi_{V}^{p} \sigma_{p}+\xi_{V}^{n}\left(\varepsilon G_{E}^{p, \gamma} G_{E}^{n, \gamma}+\tau G_{M}^{p, \gamma} G_{M}^{n, \gamma}\right)-\varepsilon^{\prime \prime} G_{M}^{p, \gamma} G_{A}^{T=1}\right. \\
\left.-\varepsilon^{\prime \prime} G_{M}^{p, \gamma} G_{A}^{T=0}\right]
\end{gathered}
$$


with

$$
\begin{gathered}
\varepsilon^{\prime \prime}=\varepsilon^{\prime}\left(1-4 \sin ^{2} \theta_{W}\right) \\
A_{L R}^{p, s}=-\frac{G_{F} Q^{2}}{\pi \alpha \sqrt{2}} \frac{1}{4 \sigma_{p}}\left[\xi_{V}^{(0)}\left(\varepsilon G_{E}^{p, \gamma} G_{E}^{s}+\tau G_{M}^{p, \gamma} G_{M}^{s}\right)-\varepsilon^{\prime \prime} \xi_{A}^{s} G_{M}^{p, \gamma} G_{A}^{s}\right]
\end{gathered}
$$

As in the proton case, we have:

$$
\begin{gathered}
A_{L R}^{n}=A_{L R}^{n, 0}+A_{L R}^{n, s} \\
A_{L R}^{n, 0}=-\frac{G_{F} Q^{2}}{\pi \alpha \sqrt{2}} \frac{1}{4 \sigma_{n}}\left[\xi_{V}^{p} \sigma_{n}+\xi_{V}^{n}\left(\varepsilon G_{E}^{p, \gamma} G_{E}^{n, \gamma}+\tau G_{M}^{p, \gamma} G_{M}^{n, \gamma}\right)+\varepsilon^{\prime \prime} G_{M}^{n, \gamma} G_{A}^{T=1}\right. \\
\left.-\varepsilon^{\prime \prime} G_{M}^{n, \gamma} G_{A}^{T=0}\right] \\
A_{L R}^{n, s}=-\frac{G_{F} Q^{2}}{\pi \alpha \sqrt{2}} \frac{1}{4 \sigma_{n}}\left[\xi_{V}^{(0)}\left(\varepsilon G_{E}^{n, \gamma} G_{E}^{s}+\tau G_{M}^{n, \gamma} G_{M}^{s}\right)-\varepsilon^{\prime \prime} \xi_{A}^{s} G_{M}^{n, \gamma} G_{A}^{s}\right]
\end{gathered}
$$

In the static approximation, the asymmetry of electron deuterium elastic scattering is given by:

$$
A_{L R}^{D}=A_{L R}^{p} \frac{\sigma_{p}}{\sigma_{p}+\sigma_{n}}+A_{L R}^{n} \frac{\sigma_{n}}{\sigma_{p}+\sigma_{n}}
$$

As in the proton and neutron cases, this deuterium asymmetry is written in the following form:

$$
\begin{aligned}
& A_{L R}^{D}=A_{L R}^{D, 0}+A_{L R}^{D, s} \\
& A_{L R}^{D, 0}=-\frac{G_{F} Q^{2}}{\pi \alpha \sqrt{2}} \frac{1}{4\left(\sigma_{p}+\sigma_{n}\right)} \\
& {\left[\xi_{V}^{p}\left(\sigma_{p}+\sigma_{n}\right)+2 \xi_{V}^{n}\left(\varepsilon G_{E}^{p, \gamma} G_{E}^{n, \gamma}+\tau G_{M}^{p, \gamma} G_{M}^{n, \gamma}\right)-\varepsilon^{\prime \prime}\left(G_{M}^{p, \gamma}-G_{M}^{n, \gamma}\right) G_{A}^{T=1}\right.} \\
& \left.-\varepsilon^{\prime \prime}\left(G_{M}^{p, \gamma}+G_{M}^{n, \gamma}\right) G_{A}^{T=0}\right] \\
& A_{L R}^{D, s}=-\frac{G_{F} Q^{2}}{\pi \alpha \sqrt{2}} \frac{1}{4\left(\sigma_{p}+\sigma_{n}\right)} \\
& {\left[\xi_{V}^{(0)} \varepsilon\left(G_{E}^{p, \gamma}+G_{E}^{n, \gamma}\right) G_{E}^{s}+\xi_{V}^{(0)} \tau\left(G_{M}^{p, \gamma}+G_{M}^{n, \gamma}\right) G_{M}^{s}-\varepsilon^{\prime \prime} \xi_{A}^{s}\left(G_{M}^{p, \gamma}+G_{M}^{n, \gamma}\right) G_{A}^{s}\right]}
\end{aligned}
$$

Let us make a few comments concerning the equations (74-83): 


\begin{tabular}{cccc}
\hline Method & $\rho_{s}$ & $\mu_{s}$ & Ref. \\
\hline Pole fits & $-2.1 \pm 1.0$ & $-0.31 \pm 0.09$ & {$[21]$} \\
& $-2.9 \pm 0.5$ & $-0.24 \pm 0.03$ & {$[22]$} \\
Kaon Loops & 0.2 & -0.03 & {$[23]$} \\
& $0.5 \pm 0.1$ & $-0.35 \pm 0.05$ & {$[24]$} \\
Unquenched Quarks & 0.3 & -0.12 & {$[25]$} \\
Meson exchange & 0.03 & 0.04 & {$[26]$} \\
Meson cloud & - & 0.002 & {$[27]$} \\
NJL & $3.0 \pm 0.08$ & $-0.15 \pm 0.10$ & {$[29]$} \\
Skyrme & 1.6 & -0.13 & {$[30]$} \\
& -0.7 & -0.05 & {$[31]$} \\
Chiral Bag Model & - & 0.37 & {$[32]$} \\
Lattice QCD & $1.7 \pm 0.7$ & $-0.36 \pm 0.20$ & {$[33]$} \\
Dispersion Relations & 0.99 & -0.42 & {$[34]$} \\
\hline
\end{tabular}

Table 2: Theoretical estimates for $\rho_{s}$ and $\mu_{s}$.

- The non strange part of the asymmetry for the proton (neutron) receives some contribution from the neutron (proton) electromagnetic form factors.

- Even if the strange form factors are equal to zero, the experimental asymmetry may have a substantial value.

The strange magnetic moment $\mu_{s}$ and the strange charge radius $\left\langle r_{s}^{2}\right\rangle$ are defined as :

$$
\begin{gathered}
<r_{s}^{2}>=-\left.6 \frac{\partial F_{1}^{s}\left(Q^{2}\right)}{\partial Q^{2}}\right|_{Q^{2}=0}, \\
\mu_{s}=\left.G_{M}^{s}(0) \quad \rho_{s} \equiv \frac{\partial G_{E}^{s}\left(Q^{2}\right)}{\partial Q^{2}}\right|_{Q^{2}=0}=-\frac{2}{3} M^{2}<r_{s}^{2}>-\mu_{s}
\end{gathered}
$$

Until now, there are no experimental constraints for $G_{E}^{(s)}$ and $G_{M}^{(s)}$. The only one theoretical constraint is that $G_{E}^{(s)}$ must vanish at $Q^{2}=0$ because the nucleon has no net strangeness. One might hope to extract informations on the strange vector current form factors by analyzing the parity violating scattering data from the measured asymmetry which is model-independent. In table2, are given some theoretical estimates for $\rho_{s}$ and $\mu_{s}$.

Many theoretical predictions (Poles fit, Kaon loops, SU(3) Skyrme ... ) for low- $Q^{2}$ behavior of $s$ quark vector current form factors give the negative value for the strange magnetic moment $\mu_{s}=G_{M}^{s}(0)$.

An issue of these parity violating experiments on the nucleon is the $Q^{2}$ or $\tau$ dependence of the strange form factors. A convenient way to describe this is to introduce new parameters [16]: 


$$
G_{E}^{s}=\rho_{s} \tau G_{D}^{V} \xi_{E}^{(s)} \quad G_{M}^{s}=\mu_{s} \tau G_{D}^{V} \xi_{M}^{(s)}
$$

with

$$
\xi_{E}^{(s)}=\frac{1}{1+\lambda_{E}^{(s)} \tau} \quad \xi_{M}^{(s)}=\frac{1}{1+\lambda_{E}^{(s)} \tau} \quad G_{D}^{V}=\frac{1}{\left(1+\frac{Q^{2}}{0.71}\right)^{2}}
$$

\section{Experimental overview}

In recent years, new $\mathrm{cw}$ electron accelerators (high intensity, high duty-factor and a polarized beam) TJNAF and MAMI, increase in a significant way, the statistical accuracy for $e p$ scattering experiments.

In this context, a fundamental issue to establishing the presence of the strange quark in the nucleon is the measurement of the strange form factors. Many experiments using polarized electron beam have been performed [35, 36, 37, 40,41] or are in progress [42, 43, 44]. There are different strategies to select the kinematic regions (forward or backward scattering), to extract the combination of the electric, magnetic and axial form factor with a reasonable accuracy.

$$
Q^{2}=0.25(\mathrm{GeV} / \mathrm{c})^{2}
$$
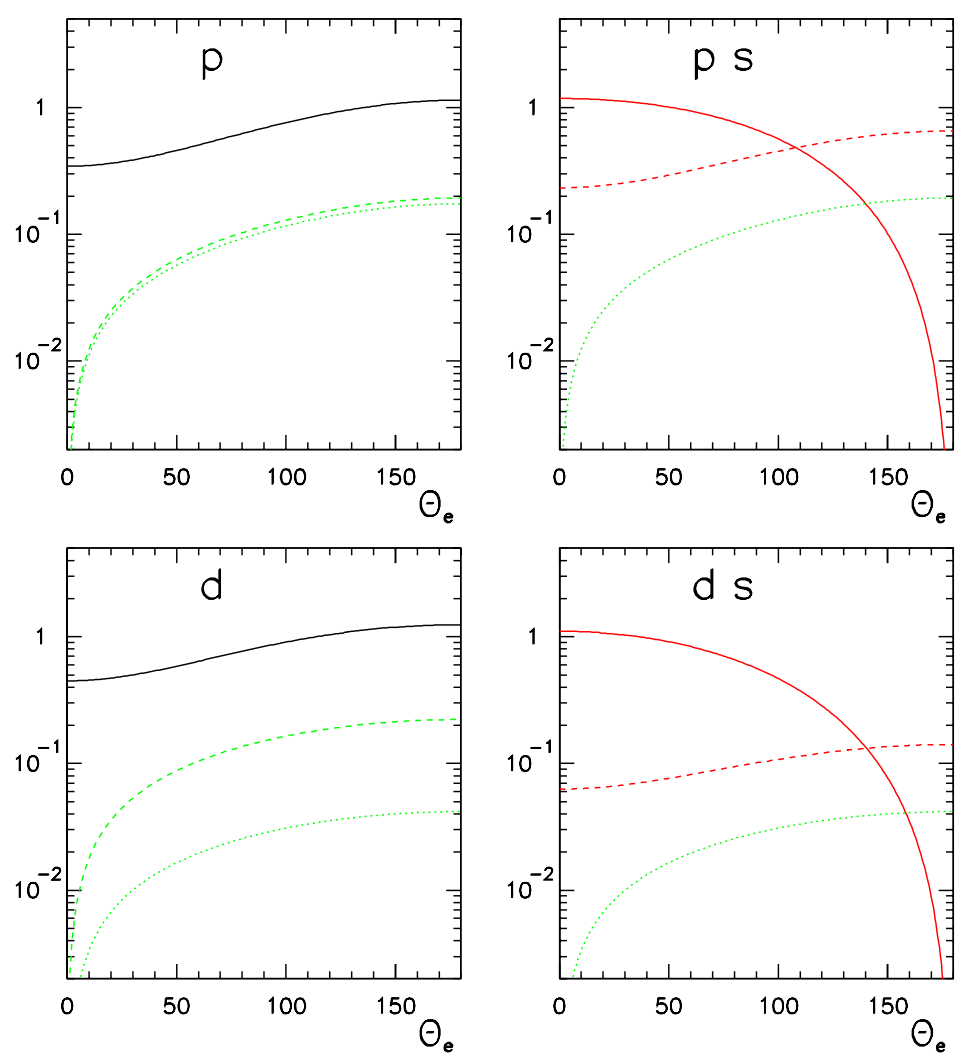

Figure 7: See Text

According to the previous section, it is possible to write the asymmetry for both the proton and the deuteron in the form: 


\begin{tabular}{ccccccc}
\hline & SAMPLE & HAPPEX & PVA4 & HAPPEX II & G0 I & G0 II \\
\hline $\mathrm{E}(\mathrm{GeV})$ & 0.2 & 3.3 & 0.855 & 3.3 & 3.0 & $0.16-0.95$ \\
$Q^{2}(\mathrm{GeV} / \mathrm{c})^{2}$ & 0.10 & 0.48 & 0.23 & 0.1 & $0.16-0.95$ & $0.16-0.95$ \\
$\varepsilon$ & 0.08 & 0.973 & 0.86 & 0.994 & 0.96 & 0.17 \\
$\langle\theta\rangle$ & 150 & 12.5 & 35. & 6 & $5-15$ & 110 \\
Particle & $\mathrm{e}$ & $\mathrm{e}$ & $\mathrm{e}$ & $\mathrm{e}$ & $\mathrm{p}$ & $\mathrm{e}$ \\
\hline
\end{tabular}

Table 3: Kinematics of proton parity-violation experiments.

$$
-\frac{4 \pi \alpha \sqrt{2}}{G_{F} Q^{2}} A_{L R}=\quad X_{1}+X_{A_{1}} G_{A}^{T=1}+X_{A_{0}} G_{A}^{T=0}+X_{E}^{s} G_{E}^{s}+X_{M}^{s} G_{M}^{s}+X_{A}^{s} G_{A}^{s}
$$

where the different coefficients $X_{1}, X_{A_{1}}, X_{A_{0}}, X_{E}^{s}, X_{M}^{s}$ and $X_{A}^{s}$ include the kinematics factors $\varepsilon, \tau$ and $\varepsilon^{\prime \prime}$ and the electromagnetic form factors using the parametrizations discussed in the previous section.

To illustrate the sensitivity of the measured asymmetry with these coefficients, we display in Fig.7 their variation as functions of the electron scattering angle for $Q^{2}=0.25(\mathrm{GeV} / \mathrm{c})^{2}$. In the left part of this figure are plotted the variation of $\left|X_{1}\right|$ (solid line), $\left|X_{A_{1}}\right|$ (dashed line) and $\left|X_{A_{0}}\right|$ (dotted line). In the right part, are plotted $\left|X_{E}^{s}\right|$ (solid line), $\left|X_{M}^{s}\right|$ (dashed line) and $\left|X_{A}^{s}\right|$ (dotted line). The upper part of the figure is related to the proton and the lower part to the deuteron.

At very forward angle, the contribution of the axial form factors should be very small and we expect to measure a combination of the electric and magnetic strange form factors. At backward angles, the contribution of the axial form factors (non strange and strange) can be substantial. In this case, with a single measurement, it will be difficult to extract the value of the electric and magnetic strange form factors $G_{E}^{s}$ and $G_{M}^{s}$. The measurements on both proton and deuterium targets is a possibility to disentangle in extracting the different form factors.

In table 3, the different kinematics of proton parity-violation experiments are displayed.

Since one is attempting to measure a small asymmetry, it is essential that the experimental conditions (beam properties) remain as identical as possible when the helicity is reversed. There are many steps taken to insure this goal. In this talk, I will not enter into the details of all the experimental techniques applied to minimize the false asymmetries which can occur.

In the following part of the section we give recent results concerning the SAMPLE and HAPPEX experiments. Let us mention two recent overview reports $[45,46]$ in which many experimental details can be found. Afterwards, we discuss the physics of PVA4 and G0, we describe the experimental set-up and give some simulation of physics backgrounds for these upcoming experiments.

\subsection{RECENT RESULTS}

\subsubsection{SAMPLE}

The SAMPLE experiment [35, 36, 37] took place at the MIT-Bates Linear Accelerator Center. It was the first experiment to determine the weak neutral magnetic form factor of the proton. The goal of this experiment was to measure $G_{M}^{s}$ at low $Q^{2}$ by elastic scattering at backward angles on hydrogen and deuterium. Elastic events were detected by the Čerenkov 
light produced as they passed through the air. An array of ten ellipsoidal mirrors focussed the Cerenkov light into ten eight-inch diameter phototubes. The beam energy of $200 \mathrm{MeV}$ was low enough so that the electrons form inelastic events were below the threshold for producing Cerenkov light in air.

The results on the hydrogen target were published in 1997 [35] and 2000 [36] with the asymmetry given in parts per million ( $\mathrm{ppm})$ :

$$
\begin{aligned}
& A=-6.34 \pm 1.45 \pm 0.53 \mathrm{ppm} \\
& A=-4.92 \pm 0.61 \pm 0.73 \mathrm{ppm}
\end{aligned}
$$

where the first uncertainty is statistical and the second is the estimated systematic error.

At the mean kinematics of the experiment, $\left(Q^{2}=0.1(\mathrm{GeV} / \mathrm{c})^{2}\right)$, the theoretical asymmetry is

$$
\mathrm{A}=-5.61+3.49 G_{M}^{s}+1.55\left[G_{A}^{T=1}\left(Q^{2}\right)+G_{A}^{T=0}\left(Q^{2}\right)+G_{A}^{s}\left(Q^{2}\right)\right]
$$

The strange magnetic form factor derived at $Q^{2}=0.1(\mathrm{GeV} / \mathrm{c})^{2}$ from the asymmetry in Eq. 90 is:

$$
G_{M}^{s}=-0.45\left[G_{A}^{T=1}\left(Q^{2}\right)+G_{A}^{T=0}\left(Q^{2}\right)+G_{A}^{s}\left(Q^{2}\right)\right]+0.20 \pm 0.17 \pm 0.21
$$

The result is displayed in Fig.8a

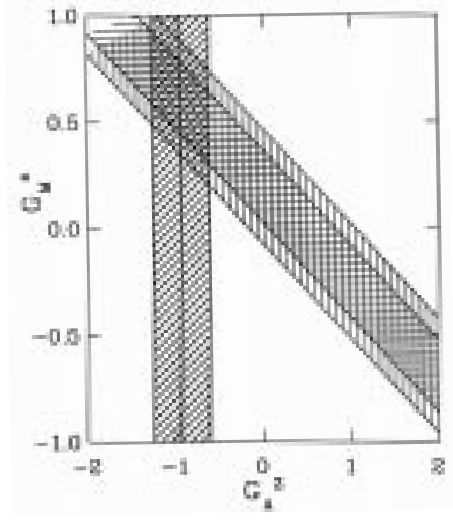

a)

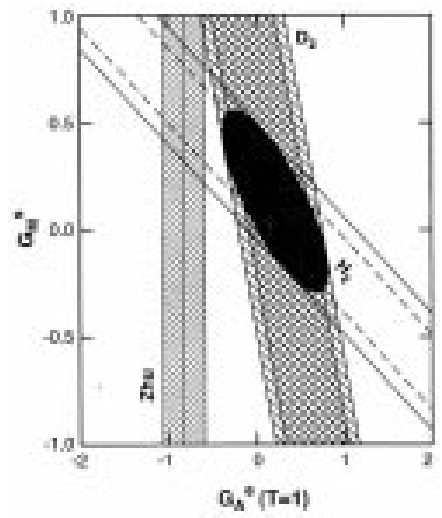

b)

Figure 8: a) Allowed region for both form factors $G_{M}^{s}$ and $G_{A}^{T=1}$. b) Combined analysis of the SAMPLE data from hydrogen and deuterium targets.

The sum of the axial form factors between square brackets including the radiative corrections [38] has been calculated and is displayed on the same Fig.8. From the experimental determination of $G_{M}^{s}$ and this calculation the authors concluded that:

$$
G_{M}^{s}\left(Q^{2}=0.1(G e V / c)^{2}\right)=+0.61 \pm 0.17 \pm 0.21 \pm 0.19
$$

This value is very large and is of the opposite sign predicted by most of theoretical models. 
To minimize the uncertainties on the radiative corrections, the asymmetry was measured on the deuterium [37]. The value is given by

$$
\mathrm{A}_{d}=[-6.79 \pm 0.64 \pm 0.55] \mathrm{ppm}
$$

In this article they give their result in terms of the isovector axial form factor:

$$
\mathrm{A}_{d}=\left[-7.27+1.78 G_{A}^{T=1}+0.75 G_{M}^{s}\right] \mathrm{ppm}
$$

for the deuterium and

$$
\mathrm{A}_{p}=\left[-5.72+1.55 G_{A}^{T=1}+3.49 G_{M}^{s}\right] \mathrm{ppm}
$$

for the proton. The combined measurement allow an independent experimental determination of $G_{M}^{s}$ and $G_{A}^{T=1}$. The result is displayed in Fig.8b) and is written:

$$
G_{M}^{s}\left(Q^{2}=0.1(G e V / c)^{2}\right)=+0.14 \pm 0.29 \pm 0.31
$$

To quote the contribution of $\mu_{s}$, they used the momentum dependence of [39]:

$$
\mu_{s}=[0.01 \pm 0.29 \pm 0.31 \pm 0.07] \mu_{N}
$$

where the last uncertainty is due to some error in the extrapolation. The authors give also an experimental result for $G_{A}^{T=1}$.

$$
G_{A}^{T=1}=+0.22 \pm 0.45 \pm 0.39
$$

This result is not compatible with the recent calculations of Zhu [20], as it is shown in Fig.8b.

\subsubsection{HAPPEX}

The HAPPEX experiment [40, 41] utilized the two spectrometers in Hall A at Jefferson Lab. to measure parity violation at $Q^{2}=0.477(\mathrm{GeV} / \mathrm{c})^{2}$ at very forward angles $(\theta=12.3 \mathrm{deg}$.). The scattered electrons were detected by integrating the output of a simple lead-scintillator calorimeter. This calorimeter was shaped to accept only the elastic electrons, which are physically well separated form the inelastic electrons in the focal plane of the polarimeter. The experiment was performed in two stages. The measured asymmetry, including the results form both phases of the experiment is:

$$
\mathrm{A}_{p}=[-14.60 \pm 0.94 \pm 0.54] \mathrm{ppm}
$$

Because the HAPPEX asymmetry was measured at forward angle, it is sensitive to $G_{E}^{s}$ and $G_{M}^{s}$ form factors (see Fig.7). The axial contribution is expected to be $-0.56 \pm 0.23 \mathrm{ppm}$, (which is small compared to $-14.6 \mathrm{ppm}$ ) assuming the calculated value for $G^{T=1}$ [20]. The other form factors enter in the combination $G_{E}^{s}+0.392 G_{M}^{s}$ for these kinematics. The value of this combination, normalized to the most accurately proton form factor, $G_{M}^{p, \gamma} / \mu_{p}$, is:

$$
\frac{G_{E}^{s}+0.392 G_{M}^{s}}{G_{M}^{p, \gamma} / \mu_{p}}=0.091 \pm 0.054 \pm 0.039
$$


We notice that the first uncertainty is a combination of the statistical and systematic uncertainties and the second is due to the uncertainty in the other electromagnetic form factors. Using the results of a different recent $G_{M}^{n, \gamma}$ measurement yields

$$
\frac{G_{E}^{s}+0.392 G_{M}^{s}}{G_{M}^{p, \gamma} / \mu_{p}}=0.146 \pm 0.054 \pm 0.047
$$

\subsection{UPCOMING EXPERIMENTS}

\subsubsection{SAMPLE and HAPPEX II}

The SAMPLE experiment will continue with a second deuterium measurement at a momentum transfer of $0.03(\mathrm{GeV} / \mathrm{c})^{2}$.

The HAPPEX group is also approved to make a measurement at $Q^{2}=0.1(\mathrm{GeV} / \mathrm{c})^{2}$. The experiment will use the same beam and target configuration of HAPPEX but at a scattering angle of $\theta_{\text {lab }}=6^{\circ}$ (HAPPEX II).

\subsubsection{PVA4}

Polarized electrons at a polarization between $75 \%$ and $80 \%$ are produced via photoeffect from strained layer Ga-As-Crystals. The electrons are then accelerated in the Mainz Microtron accelerator (MAMI) to an energy of $0.855 \mathrm{GeV}$. The electron then hits the high cooling power, high flow liquid hydrogen target. The energy of the scattered particles is then measured in a absorbing $\mathrm{PbF}_{2}$ Cerenkov shower calorimeter. The Luminosity is measured under small angles behind the target.
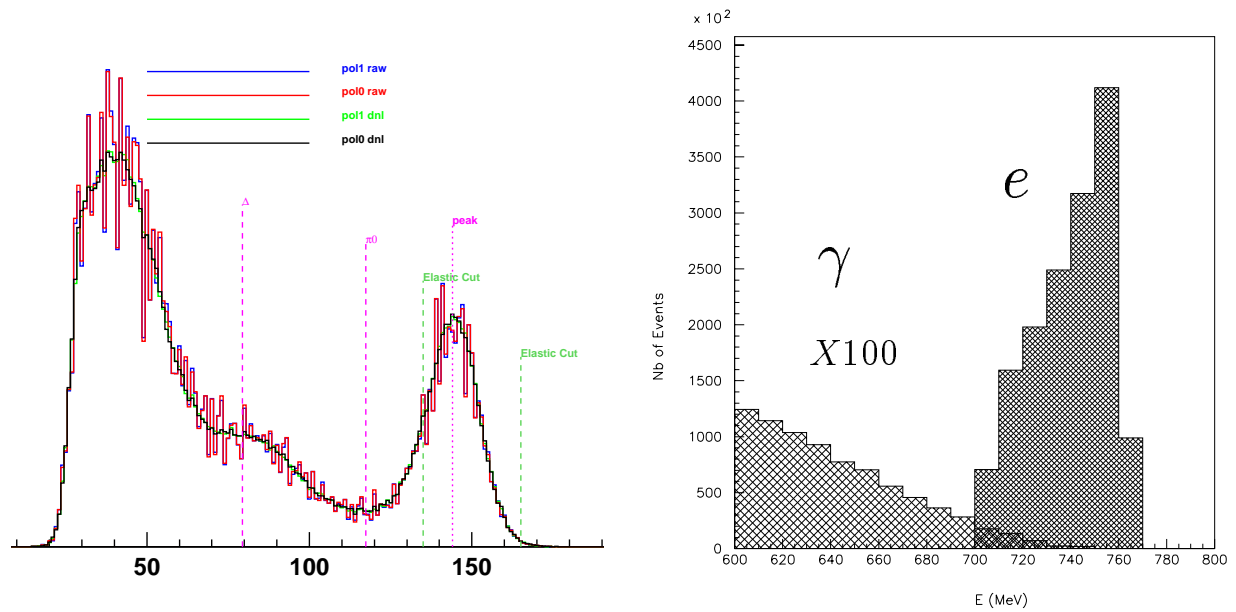

Figure 9: Typical histogram in the PVA4 experiment (left) and background due to $\pi^{0}$ decay in two photons (right).

The $\mathrm{PbF}_{2}$ Cerenkov shower calorimeter consists presently of 511 single crystals. It covers a scattering angle from $30^{\circ}$ to $40^{\circ}$. The PVA4 experiment [42] will measure the linear combination $G_{E}^{s}+0.22 G_{M}^{s}+0.04 G_{A}^{T=1}$. A typical histogram is shown in Fig.9. This spectrum shows a large elastic peak with some additional events above the $\pi$ threshold. Without 
taking into account of the response of the detector, the study of this background has been performed in the angular range $30^{\circ} \leq \theta_{e} \leq 40^{\circ}[47]$. Besides the contribution of the elastic radiative tail, the photons coming from high energy $\pi^{0}$ decay contribute to this background. The rate, which is small as compared to the elastic peak, is shown in Fig.9

\subsubsection{G0}

The goal of the G0 experiment [43] is to measure forward proton asymmetries for $0.12 \leq$ $Q^{2} \leq 1 .(\mathrm{GeV} / \mathrm{c})^{2}$ and backward asymmetries for both the proton and the deuteron for $Q^{2}=$ $0.2,0.5$ and $0.8(\mathrm{GeV} / \mathrm{c})^{2}$. From this complete set of observables we expect to determine the charge, magnetic and axial weak currents of the nucleon. The G0 experiment will use a $40 \mu \mathrm{A}, 70 \%$ polarized beam from the Jlab accelerator.

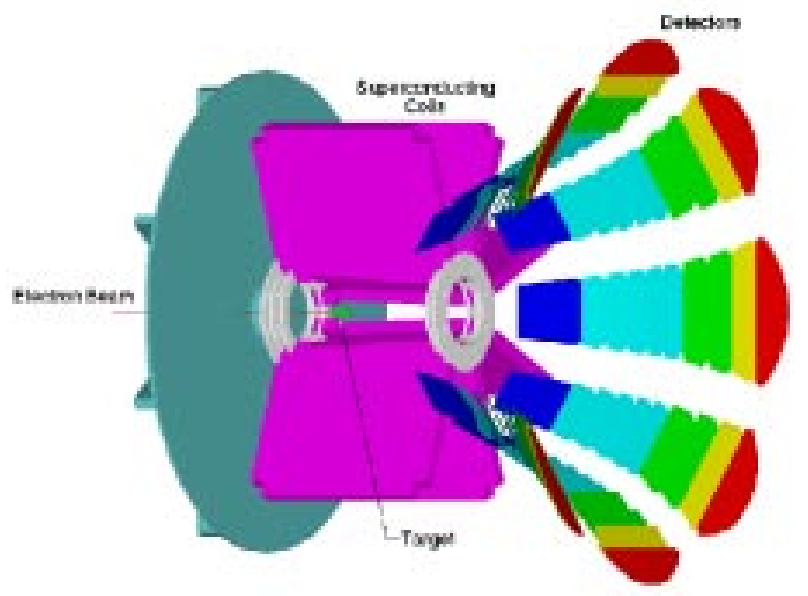

Figure 10: Schematic of the G0 parity-violating electron scattering experiment.
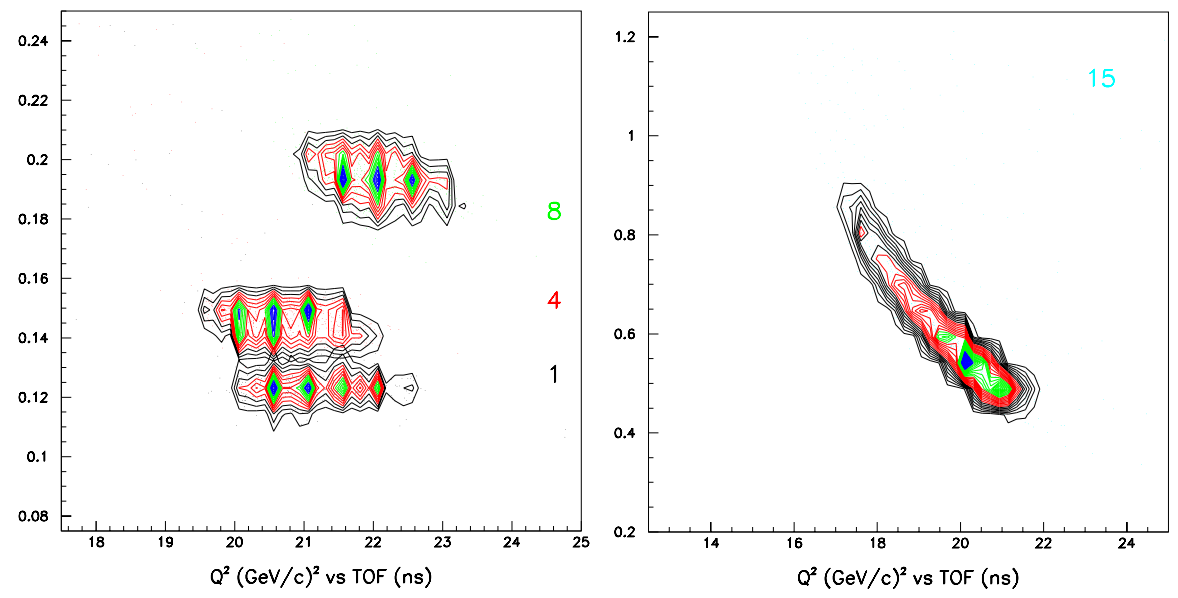

Figure 11: $Q^{2}$ dependence with TOF. Left: Detectors 1,4,8. Right:Detector 15

The experimental apparatus consists of a superconducting toroidal magnet to focus particles from a $20 \mathrm{~cm}$ liquid hydrogen target to an array of plastic scintillator pairs (Fig.10).

In the phase I, forward angle asymmetries will be measured by detecting the recoil protons from elastic scattering. The scattered events $\left(62^{\circ} \leq \theta_{p} \leq 78^{\circ}\right)$ correspond to $15^{\circ} \leq \theta_{e} \leq 5^{\circ}$. The entire $Q^{2}$ range is obtained simultaneously with an incident beam energy of $3 \mathrm{GeV}$ (see Fig.11) 

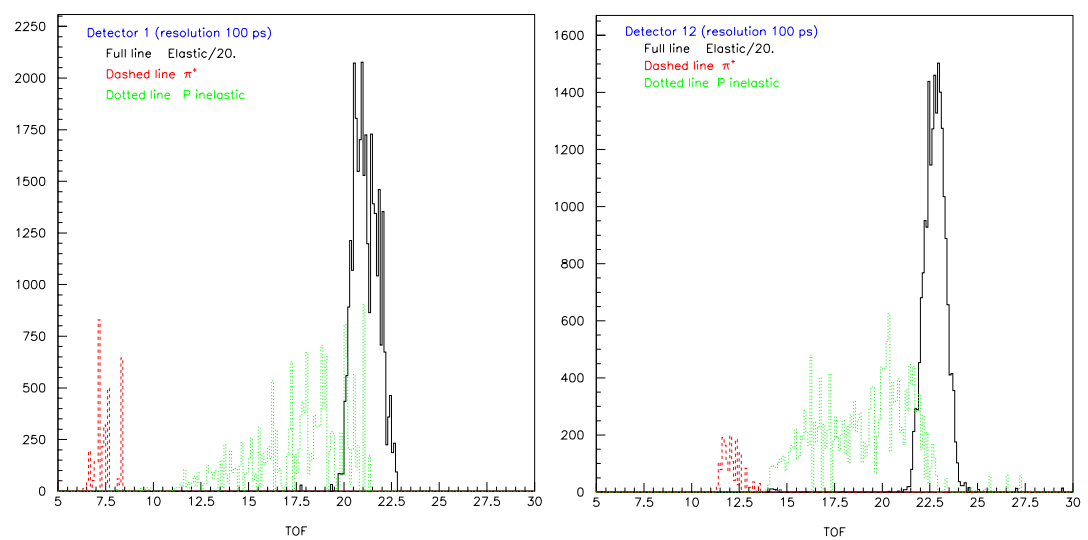

Figure 12: Counting rate versus Time Of Flight. Black line: Elastic proton/20. Red line: $\pi^{+}$. Green line: Inelastic proton. Left: Detector number 1. Right: Detector number 12
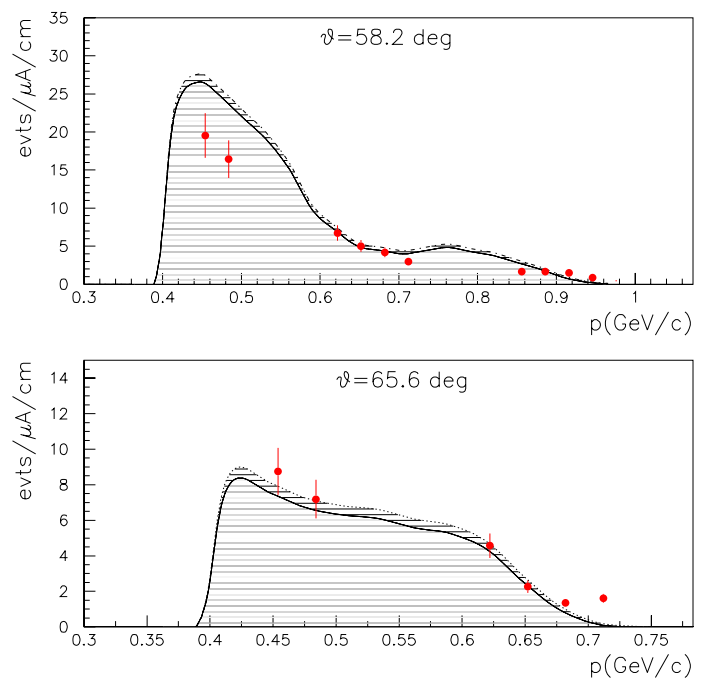

Figure 13: Comparison between the SOS data and the simulation result.

For this measurement, elastic protons are identified by Time-Of-Flight. The other particles such as $\pi^{+}$and strongly inelastic protons are rejected, as it is shown in Fig.12.

As the proton is heavy compared to the electron, the maximum energy of the proton in the inelastic channels is not very different (at least for 1 pion electro-production) from the energy in the elastic channel.

Our colleagues from Grenoble [48] have shown in a dedicated experiment (with the SOS spectrometer) that for the strongly inelastic protons, the most part of the background comes from the photoproduction of one or more pions due to the bremsstrahlung photons (see Fig.13).

For the inelastic protons with momentum close to the elastic events, we need an accurate generator for inclusive electroproduction reactions.

In the energy range $\left(0.2 \leq E_{\gamma^{*}} \leq 3 . \mathrm{GeV}\right)$ of forward $\mathrm{G} 0$, there is no reliable theory model to predict such cross-sections. At electron energy lower than $1 \mathrm{GeV}$, where effective lagrangians are good and are able to reproduce such inclusive cross-sections [49], we have shown that using the photo-production cross-sections with a virtual flux is a very good approximation. Extending this assumption in our energy range, we have developed a model to calculate the proton inclusive cross-sections [50] using the photo-production data [51]. In 


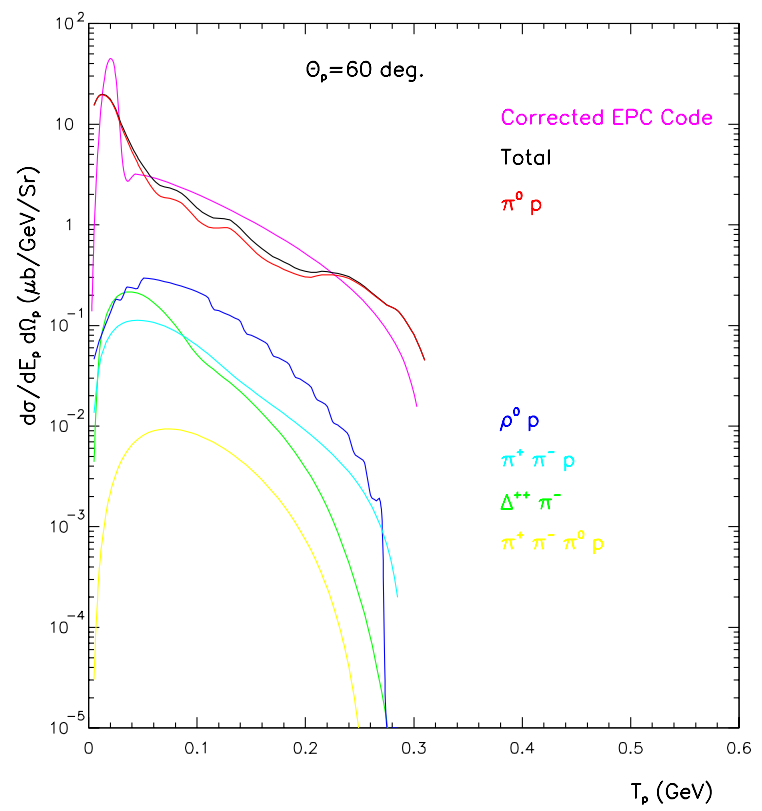

Figure 14: Contribution of different channels in the Orsay Code [50] and comparison with the corrected EPC code.

this calculation, we have taken into account the cross-sections of several channels ( one pion, two pions,... ) (see Fig.14).

In the phase II, the electrons scattered on hydrogen and deuterium targets will be detected at backward angle $\left(\theta_{e} \simeq 110^{\circ}\right)$. The energy of the electron beam $\left(0.330 \leq E_{e} \leq 0.950\right.$ $\mathrm{GeV})$ will be adjusted to reach the values of $0.2,0.5$ and $0.8(\mathrm{GeV} / \mathrm{c})^{2}$ for $Q^{2}$. The backward detection will require improved particle identification to separate electrons and $\pi^{-}$(mainly in the deuterium measurements).

\section{Summary and conclusion}

The experimental program of studying parity violation in polarized electron scattering will provide new and exciting informations towards a complete understanding of nucleon structure in the framework of QCD. The forthcoming data from PVA4, HappexII and G0 would have the sensitivity to establish the presence of the strange quark in the nucleon.

Considerable effort was devoted to control the systematic errors in the measured asymmetry and the progress realized in the experimental techniques will open a promising domain of high precision experiments. Parity violating left-right asymmetries have played important role for a better understanding of the Standard Model and also provided a cleanest probe of new physics (compositeness, another weak boson $Z^{\prime}$, anomalous anapole moment ... ).

In this context, there are two future experiments proposed at SLAC (E158) [52] and TJNAF(PAC 19) [53]. E158 scheduled to run at SLAC in 2001, will measure the asymmetry in Moller scattering. The predicted asymmetry is $0.32 \mathrm{ppm}$ and the goal of the experiment is the measurement of $\sin ^{2}\left(\theta_{W}\right)$ with a precision of \pm 0.0008 , compare to the value of \pm 0.0002 from SLD and LEP.

The second plans to measure the isovector parity-violating pion-nucleon coupling $h_{\pi N N}^{(1)}$. 
This can be done by measuring the PV asymmetry in $\pi^{+}$photoproduction from a proton target near threshold and the predicted asymmetry is about $0.2 \mathrm{ppm}$.

\section{Acknowledgments}

The authors are grateful to J. Arvieux, R. Frascaria, F. Maas, D. Marchand, M. Morlet, J-S. Réal and R. Tieulent for their help in preparing this manuscript. We thank the G0 and PVA4 Collaboration members who work so hard to bring that projects to their present states. One of us (J. VdW) would like to thank the organizers of this very fruitful conference.

\section{References}

[1] S. Okubo, Phys. Lett. 5, 165 (1963); G. Zweig, CERN preprints 401,412 (1964) unpublished; J. Iizuki, Suppl. Prog. Theor. Phys. 37, 21 (1966).

[2] J.F. Donoghue and C.R. Nappi, Phys. Lett. B 168, 105 (1986).

[3] D. Kaplan and A. Manohar, Nucl. Phys. B 310, 527 (1988).

[4] J. Ashman et al., Phys. Lett. B 206, 364 (1988); Nucl. Phys. B 328, 1 (1989).

[5] HERMES Collaboration, A. Airapetian et al., Phys. Rev. Lett. 84, 4047 (2000); hepex/0104005.

[6] COMPASS Collaboration, CERN/SPSLC 96-14, SPSLC/P297 (1996).

[7] C.Y. Prescott et al., Phys. Lett. B 77, 347 (1978); B 84, 524 (1979).

[8] F. Halzen and A.D. Martin, Quarks and leptons, Wiley (1984).

[9] P.E. Bosted, Phys. Rev. C 51, 409 (1995), and references therein.

[10] H. Anklin et al., Phys. Lett. B 336, 313 (1994).

[11] P. Markowitz et al., Phys. Rev. C 48,R5 (1993); H. Gao et al., Phys. Rev. C 50, R546 (1994).

[12] E. Bruins et al., Phys. Rev. Lett. 75, 21 (1994).

[13] C. Jones et al., Phys. Rev. C 44, R571 (1991); A. Thompson et al., Phys. Rev. Lett. 68, 2901 (1992); T. Eden et al., Phys. Rev. C 50, R1749 (1994).

[14] M. Meyerhoff et al., Phys. Lett. B 327, 201 (1994); C. Herberg et al., Eur. Phys. J. A 5, 131 (1999).

[15] S. Galster et al., Nucl. Phys. B 32, 221 (1971).

[16] M.J. Musolf et al., Phys. Rep. 239, 1 (1994).

[17] Particle Data Group, Euro. Phys. J. C 54, 1 (2000).

[18] R.L. Jaffe and A.V. Manohar, Nucl. Phys. B 337, 509 (1990).

[19] H. Lipkin and M. Karliner, Phys. Lett. B 461, 280 (1999).

[20] Shi-Lin Zhu, S. J. Puglia, B. R. Holstein, and M.J. Ramsey-Musolf Phys. Rev. D 62, $033008(2000)$. 
[21] R.L. Jaffe, Phys. Lett. B 229, 275 (1989).

[22] H.W. Hammer, Ulf-G. Meissner and D. Drechsel, Phys. Lett. B 367, 323 (1996).

[23] W. Koepf, E.M. Henley and S.J. Pollock, Phys. Lett. B 288, 11 (1992).

[24] M.J. Musolf and M. Burkhardt, Z. Phys. C 61, 433 (1994).

[25] H. Ito, Phys. Rev. C 52, R1750 (1995).

[26] P. Geiger and N. Isgur, Phys. Rev. D 55, 299 (1997).

[27] Ulf-G. Meissner et al., Phys. Lett. B 408, 381 (1997).

[28] B.-Q Ma, Phys. Lett.. B 408, 387 (1997).

[29] H. Weigel et al., Phys. Lett. B 353, 20 (1995).

[30] N.W. Park, J. Schechter and H. Weigel, Phys. Rev. D 43, 869 (1991).

[31] N.W. Park and H. Weigel, Nucl. Phys. A 541, 453 (1992).

[32] S-T. Hong, B-Y. Park and D-P. Min, Phys. Lett. B 414, 229 (1997).

[33] S.J. Dong, K.F. Liu and A.G. Williams, Phys. Rev. D 58, 074504 (1998).

[34] H.W. Hammer and M.J. Ramsey-Musolf, Phys. Rev. C 60, 045205 (1999).

[35] B. Mueller et al., Phys. Rev. Lett. 78, 3824 (1997)

[36] D.T. Spayde et al., Phys. Rev. Lett. 84, 1106 (2000).

[37] R. Hasty et al. Science 290, 2117 (2000).

[38] M.J. Musolf and B.R. Holstein, Phys. Lett. B 242, 461 (1990).

[39] T.R. Hemmer, U.G. Meissner, S. Steininger, Phys. Lett. B 437, 184 (1998).

[40] K.A. Aniol, et al (HAPPEX Collaboration), Phys. Rev. Lett. 82, 1096 (1999).

[41] K.A. Aniol, et al (HAPPEX Collaboration), nucl-ex/00060002.

[42] Mainz experiment PVA4. D.von Harrach, spokeperson; F. Maas, contact person.

[43] Jefferson Lab experiment 00-006, D. Beck, spokesperson. 383 (1996).

[44] Jlab experiment 99-115 (K.S. Kumar and D. Lhuillier, spokespersons).

[45] K.S. Kumar and P.A. Souder, Progress in Particle and Nuclear physics 45, S333-S395 (2000)

[46] D.H. Beck and R.D. McKeown, hep-ph/0102334

[47] S. Ong, M. P. Rekalo, J. Van de Wiele, Eur. Phys. J. A 6, 215 (1999).

[48] R. Tieulent and J-S. Réal, G0 Internal report, G0-00-019 (2000)

[49] S. Ong and J. Van de Wiele, Phys. Rev. C 63, 024614 (2001).

[50] M. Morlet, S. Ong and J. Van de Wiele, Internal Report, IPNO DR 01-015, Orsay July 9, 2001.

[51] P. Corvisiero et al. NIM A 346, 433 (1994)

[52] SLAC experiment E158, K.S. Kumar, spokesperson, E.W. Hughes and P.A. Souder, deputy spokepersons.

[53] Proposal to TJNAF PAC 19, R. Suleiman, spokeperson. 